\title{
The physics of galaxy evolution with EAGLE
}

\author{
M. Puech ${ }^{1, a}$, M. Lehnert ${ }^{1}$, Y. Yang ${ }^{1}$, J.-G. Cuby ${ }^{2}$, S. Morris ${ }^{3}$, C. Evans ${ }^{4}$, B. Neichel ${ }^{5,1,6}$, T. Fusco ${ }^{5}$, G. \\ Rousset $^{7}$, and H. Flores ${ }^{1}$ \\ 1 GEPI, Observatoire de Paris, 5 Place Jules Janssen, 92195 Meudon Cedex, France \\ 2 LAM, OAMP, 38 rue Frederic Joliot Curie, 13388 Marseille Cedex 13, Francee \\ 3 Department of Physics, Durham University, South Road, Durham, DH1 3LE, UK \\ 4 UK ATC, Royal Observatory Edinburgh, Blackford Hill, Edinburgh, EH9 3HJ, UK \\ 5 ONERA, BP 72, 92322 Chatillon Cedex, France \\ 6 Gemini Observatory, Colina El Pino s/n, Castilla 603, La Serena, Chile \\ 7 LESIA, Observatoire de Paris, 5 Place Jules Janssen, 92195 Meudon Cedex, France
}

\begin{abstract}
One of the prominent science goal of the ELTs will be to study the physics and mass assembly of galaxies at very high redshifts. Here, we present the galaxy evolution science case for EAGLE, which is a NIR multi-integral field spectrograph for the E-ELT currently under phase A study. We summarize results of simulations conducted to derive high-level requirements. In particular, we show how we have derived the specifications for the ensquared energy that the AO system needs to provide to reach the scientific goals of the instrument. Finally, we present future strategies to conduct galaxy surveys with EAGLE.
\end{abstract}

\section{Introduction}

EAGLE is a multi-IFU, near-IR spectrograph assisted by Multi-Object Adaptive Optics (MOAO) for the E-ELT. It is a French-UK partnership currently under a phase A (design study) in collaboration with ESO. The current conceptual design is presented in detail elsewhere in these proceedings [1$3]$. Briefly, EAGLE has a patrol field of with an equivalent diameter of 7-arcmin, within which 20 , $1.65 \times 1.65 \operatorname{arcsec}^{2}$ FoV IFUs can be deployed. Image slicers will provide 37.5 mas slices. EAGLE will cover from 0.8 to $2.5 \mu \mathrm{m}$ at a spectral resolution of 4000 in its low resolution, or 10000 in its high resolution mode. A MOAO (Multi-Object Adaptive Optics) system will deliver excellent image quality using an array of up to 6 LGS and 5 NGS, correcting for atmospheric turbulence using deformable mirrors within the instrument and the large deformable mirror in the telescope itself. An on-sky demonstrator, called CANARY, is under development in parallel to the EAGLE study $[4,5]$.

The conceptual design is driven by five top-level science cases:

- Physics and evolution of high-redshift galaxies

- Detection and characterisation of 'first light' galaxies

- Galaxy assembly and evolution from stellar archaeology

- Star-formation, stellar clusters and the initial mass function

- Co-ordinated growth of black holes and galaxies

Here, we focus on the first science case. A summary of EAGLE capabilities relative to the second science case is given by Evans et al. [1], while Paumard et al. illustrate the E-ELT capabilities relative to the two last cases [6].

\section{Understanding galaxy evolution with EAGLE}

One of the main challanges in extragalactic astronomy is to understand how galaxies formed and evolved. One of the main issues is to trace mass assembly in galaxies as a function of cosmic time.

\footnotetext{
a e-mail: mathieu.puech@obspm.fr
} the original work is properly cited. 

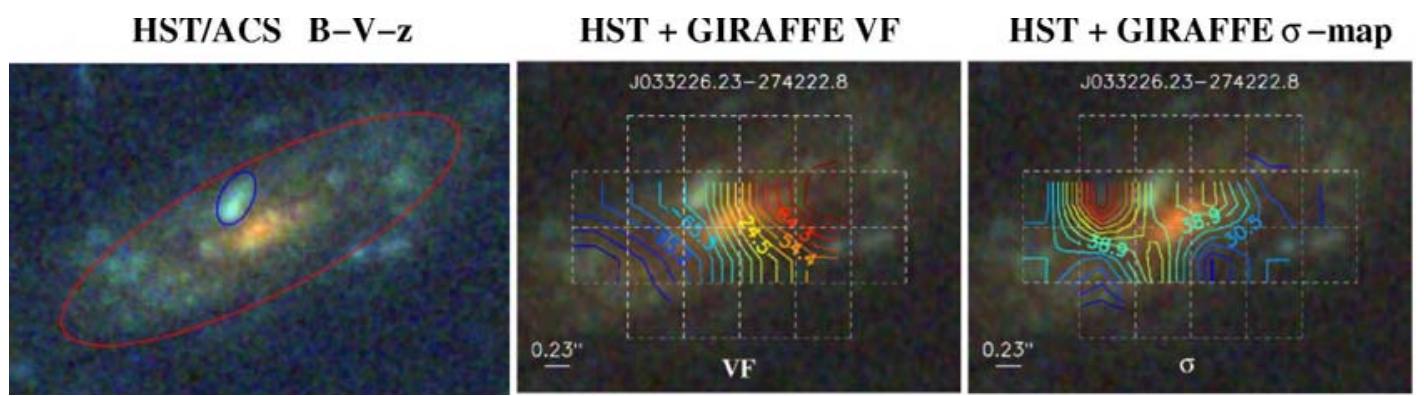

Fig. 1. 3D spectroscopy of a minor merger occuring in a $\mathrm{z} \sim 0.6$ star-forming galaxy. From left to right: HST/ACS $B V z$ color image. The red ellipse marks the main progenitor, while the blue ellipses indicates the infalling satellite; GIRAFFE velocity fields supersimposed in the HST color image; GIRAFFE velocity dispersion map superimposed on the HST color image. The white dash-lines in the two last panels represent the GIRAFFE IFU pixels.

Different physical mechanisms are known to drive this evolution. Major mergers are collisions between galaxies of similar mass, which can strongly enhance the conversion of gas into stars in the two progenitors. Another channel of driving star formation is through minor mergers, which are galaxy collisions between progenitors with mass ratio of less than 1:3. Therefore, these minor collisions individually have a much weaker effect on the mass assembly of a given galaxy, but they are thought to occur more frequently over its lifetime. Another channel is direct cold gas accretion from intergalactic filaments. We do not understand yet what is the dominant channel as a function of mass and time.

3D spectroscopy can now routinely derive the spatially-resolved kinematics of massive distant galaxies, up to $\mathrm{z} \sim 3$. This has allowed us to make a major breakthrough in our understanding of distant galaxies, and in particular of what drives star formation in distant objects. In Fig. 1, we show one example of how 3D spectroscopy can help in disentangling various physical processes. This $H S T / A C S$ color image (on the left) reveals a blue elongated region (see the blue ellipse) superimposed on a $\mathrm{z} \sim 0.6$ galaxy. The kinematic maps (obtained using GIRAFFE at the VLT, a high spectral resolution optical spectrograph with multi-IFUs amongst other capabilities [7]) do not show any particular perturbation in the velocity field of this galaxy, which seems to be a normal rotating spiral galaxy. On the contrary, the velocity dispersion map is not what is expected from a regular unperturbed rotating galaxy, since its peak of dispersion (see the red isocontours) is off-centered compared to the dynamical centre. This peak falls very close to the blue elongated region seen on the $H S T$ color image. These observations are exactly what is expected form a minor merger occuring in a rotating disk: the gas expelled from the infalling satellite during the merging produces shocks when it encounters the gas contained in the main progenitor. The off-centered peak of the velocity dispersion is a kinematic signature of this process.

These observations illustrate how 3D spectroscopy is a quantitative tool when one wants to disentangle physical processes driving star formation in distant galaxies. By mapping the physical and chemical properties of galaxies all the way up to $\mathrm{z} \sim 6$, EAGLE will the best tool for studying mass assembly in distant galaxies.

\section{Determining the requirements for the EAGLE IFUs}

The main difficulty in defining the characteristics of an IFU for EAGLE is that all the physical processes that one wants to study are operating on very different spatial scales. As shown in Fig. 1, minor mergers lead to spatial signatures on the kpc scale. Major mergers are more violent processes that can destroy orbital motions, i.e., rotation in disks, which therefore have spatial signatures on larger scales $(\sim 10-100 \mathrm{kpc})$. On the other hand, cold gas accretion can feed galaxies with fresh gas on different scales, depending on mass and redshift. To elucidate these processes, one has to adapt the "scalecoupling" of the IFU. The scale-coupling is the ratio between the spatial scale to be resolved by the IFU (i.e., galaxy diameter for major mergers), to the size of the spatial element of resolution. In most cases, MOAO will deliver a PSF with a FWHM smaller than two IFU spaxels. This means that the 


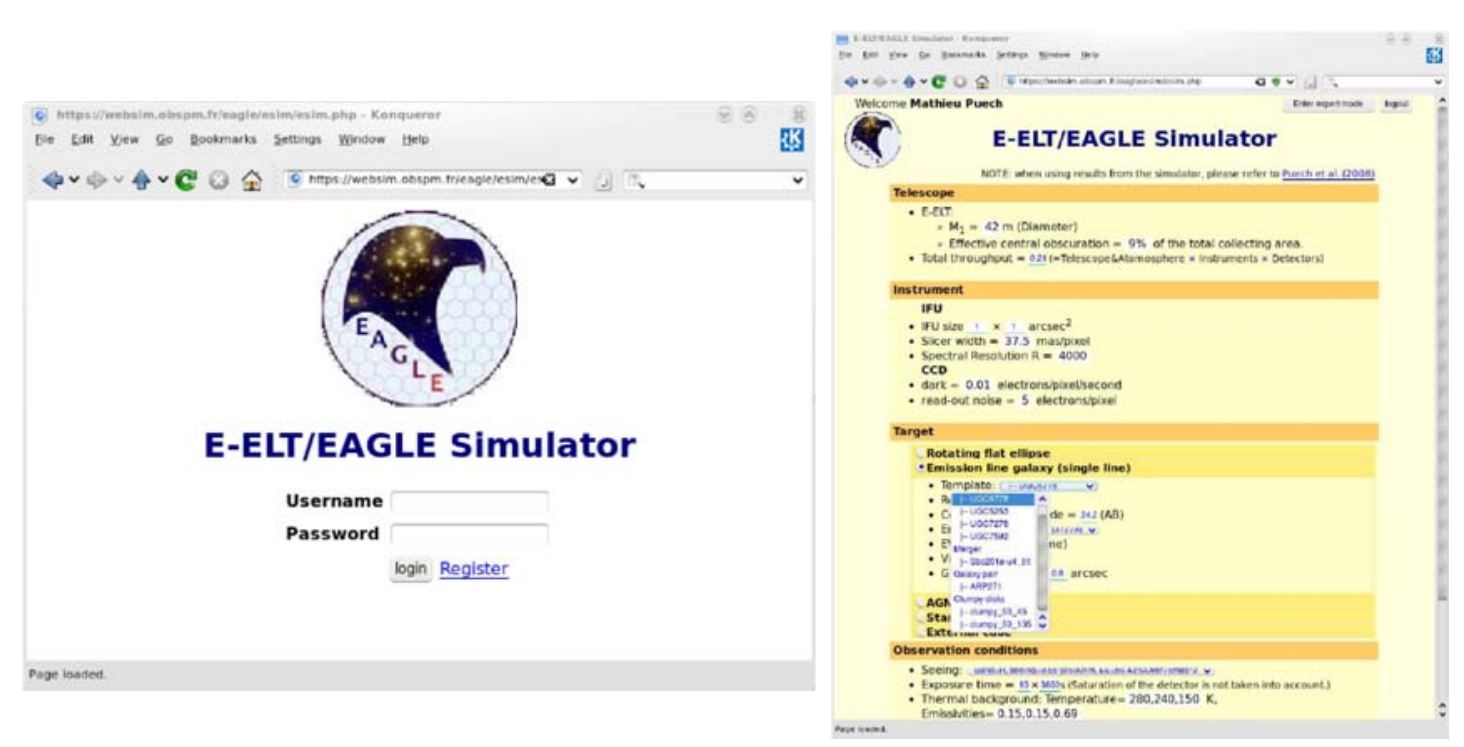

Fig. 2. Web pages of the EAGLE simulator. The user can pick up a science case using a menu (sed right-hand panel) and modify the parameters corrsponding to the telescope, instrument, object, sky, and detector, as listed.

element of spatial resolution is almost always driven by the spaxel size (i.e., two spaxels), and not directly by the PSF [9].

The scale-coupling directly drives the precision on the physical quantity one wants to study. For instance, a modest scale-coupling is already enough to recover the rotation velocity of distant galaxies, while a much finer spatial resolution is needed if one wants to recover the whole shape of the rotation curve [10], which is needed to study mass profiles. Moreover, once one has decided what is the optimal scale-coupling, one has to take care of the cross-talk induced by the PSF wings. Indeed, AO corrections leave a residual halo sourrounding a diffraction-limited core, which results in a mixing of the spectral information coming from adjacent spectra on the detector. Therefore, it is also important to characterize what level of PSF contrast one needs to recover a specific physical quantity, which directly implies how easy and accurate it is to distinguish discrete features over the spatial sampling [9]. Both the scale-coupling and the contrast can be parameterised using the Ensquared Energy (EE), which is defined as the energy of the PSF entering one element of spatial resolution.

Determining the optimal scale and EE for the EAGLE IFU is not a straighforward task. We have therefore developed a tool which allows us to simulate observations of distant galaxies in a very realistic way. This simulation tool, with web interface, allows the EAGLE science team to log-on and run simulations to constrain the requirements of their specific science case (see Fig. 2). Details about the simulations pipeline can be found elsewhere $[9,11]$. This is a modified version of the pipeline used to conduct the design reference mission of the E-ELT for the galaxy evolution science case [12]. This pipeline produces simulated EAGLE datacubes that can then be analyzed using the same tools as for real data. From these scientific products (e.g., a velocity field), one can assess what is, e.g., the required sampling, or the transmission required to reach a given scientific goal.

\section{What is the relevant spatial scale for studying galaxy evolution?}

In Fig. 3, we show a histogram of the size of HII regions in local galaxies from Elmegreen et al. [13]. The E-ELT will not be able to resolve individual HII regions in very distant objects ( $\mathrm{z} \sim 2$ and farther), with only the largest HII complexes resolved. What then is the optimal spatial scale for studying star formation in distant galaxies?

$H S T$ images of cosmological fields have revealed that the morphology of distant galaxies is much more complex than in the local Universe. In particular, kpc-sized clumps appear to be ubiquitous 


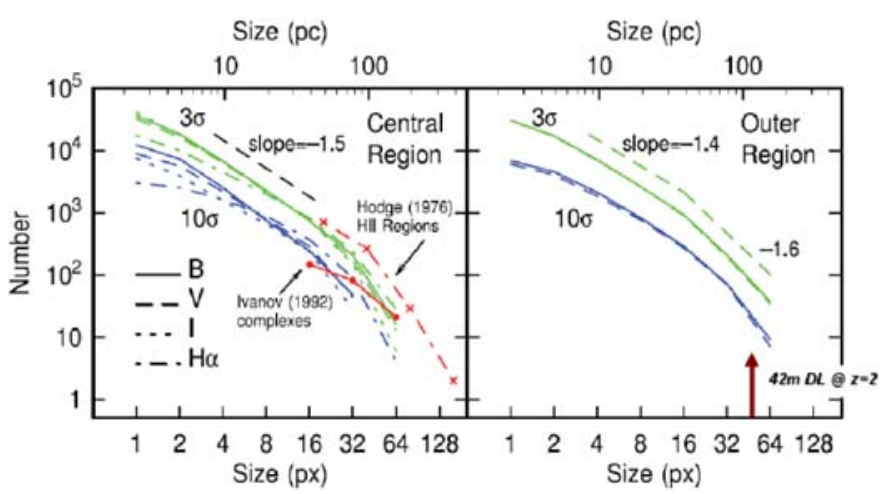

Fig. 3. Histograms of HII regions in the local galaxy NGC 628, both in the central and outer regions [13]. The red arrow in the bottom of the right panel indicates the limit of diffraction of the E-ELT at $\mathrm{z}=2$.

in galaxies at $\mathrm{z}>1$ [14]. These clumps have sizes of 120-160 mas over the redshift range 1-5, which means that an EAGLE slice width of $37.5 \mathrm{mas}$ is particularly well-suited to study these clumps, offering a scale-coupling of 3-4. Such a coupling will allow us to detect rotation in these clumps, which is crucial to understand whether these clumps are a result of Jeans-instabilites in distant gas-rich disks fed by cold streams. Indeed, recent numerical simulations have shown how this process could be an important channel for the formation of bulges and disks $[15,16]$.

\section{Simulations of distant galaxies: Specifications for the EE}

Using the simulation pipeline, we obtained simulated kinematic maps of a $\mathrm{z}=4$ Jeans-unstable disk, a merging pair of Sbc galaxies, and a regular rotating disk ${ }^{1}$. The last cases were used to derive the optimal scale and EE required to distinguish a major merger of a regular rotation. This case is discussed in detail elsewhere [9]. Here, we focus on the simulations of the Jeans-unstable clumpy disk, which is shown in Fig. 4. From these simulations, one can see that clumps become detectable with at least $\sim 20 \%$ of EE in 75 mas, while $30 \%$ provides us with a more robust measurement in case of limited signal-to-noise ratio.

The resulting EE specifications for EAGLE, as a function of a given scientific goal, are summarized in Tab. 1.

Table 1. Constraints on the EE derived from simulations, depending on the scientific objective.

\begin{tabular}{|c|c|c|c|}
\hline Spatial scale & Minimal EE & Optimal EE & Comments \\
\hline Large-scale motions & $\begin{array}{c}30 \% \text { in } 150 \mathrm{mas} \\
(\sim 14 \% \text { in } 75 \mathrm{mas})\end{array}$ & $\begin{array}{c}30 \% \text { in } 100 \mathrm{mas} \\
(\sim 25 \% \text { in } 75 \mathrm{mas})\end{array}$ & Good confidence level \\
\hline Clump detection & $20 \%$ in $75 \mathrm{mas}$ & $30 \%$ in $75 \mathrm{mas}$ & Structure of clumps uncertain \\
\hline
\end{tabular}

Finally, a trade-off was made between the two science goals, taking account on the confidence level of all constraints, which is presented in Tab. 2. This table justifies why the current baseline for EAGLE assumes $30 \%$ of EE in 75 mas.

\footnotetext{
1 We are especially indebted to T. J. Cox and F. Bournaud who provided us with the hydro-dynamical simulations of merging galaxies and clumpy disks respectively, and to P. Amram and B. Epinat who have provided us with kinematical data of local galaxies from the GHASP survey.
} 


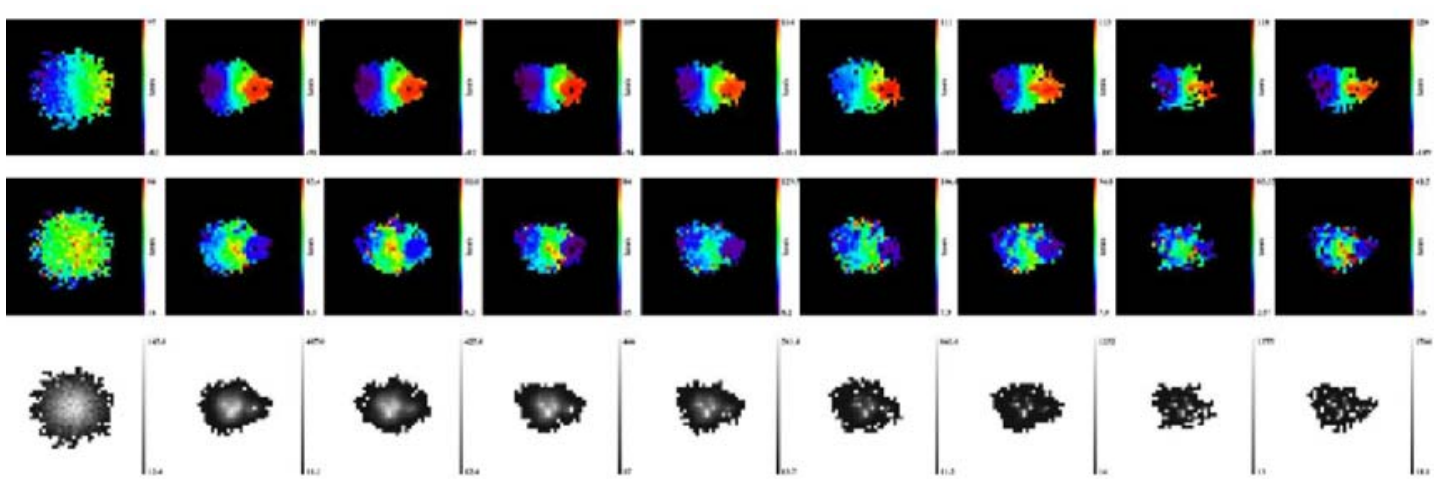

Fig. 4. Simulations of a clumpy disk at $\mathrm{z}=4$. From top to bottom: velocity field in the gas (approaching motions are in blue, while receding motions are in red), velocity dispersion map, and [OII] emission line map. The different columns correspond to increasing EE. The EE in 75 mas is $15 \%$ for the second column, while it is $61 \%$ in the last one. The first column shows the seeing-limited case for comparison. In all simulations, the pixel scale is 37.5 mas, and the spectral resolution is 4000 . Images are $0.8 \times 0.8 \operatorname{arcsec}^{2}$. These simulations were run with a conservative Equivalent Width $\mathrm{EQW}=30 \AA$. It is therefore a challenging experiment to retrieve clumps in these galaxies, since currently observed galaxies at $\mathrm{z} \sim 2$ in such objects have $\mathrm{EQW} \sim 100 \AA$.

Table 2. EE Specifications for EAGLE.

\begin{tabular}{|c|c|c|c|}
\hline & Minimal & Optimal & Goal \\
\hline EE required in 75 mas & $15 \%$ & $25 \%$ & $30 \%$ \\
\hline
\end{tabular}

\section{Conducting surveys of high-z galaxies with EAGLE}

Understanding the physical mechanisms driving galaxy evolution will require 3D spectroscopy of a representative sample of galaxies at different redshifts. So far, only two such samples have been studied extensively with 3D spectroscopy: the IMAGES sample, with 100 galaxies at $0.4<\mathrm{z}<1 \mathrm{using}$ FLAMES/GIRAFFE at the VLT [17], and the SINS sample, with 63 galaxies at $1.3<\mathrm{z}<2.6$, using VLT/SINFONI [18]. Both samples are thought to be approximately representative of galaxies more massive than $\sim 10^{10} \mathrm{M}_{\odot}$. Other samples at $2<\mathrm{z}<3$ and $\mathrm{z} \sim 1.6$ were studied using OSIRIS on the Keck telescope $[19,20]$, but they are drastically limited in size and/or whether they are representative in their distribution of stellar mass remains unclear.

Such samples clearly allowed us to make an impressive leap forward into our understanding of galaxy evolution and formation. However, we are already reaching the limits of what can be done on a $8-10 \mathrm{~m}$ telescopes, even equipped by state-of-the-art adaptive optics systems. This is particularly true at $\mathrm{z}>1$, where emission lines are observed in the NIR H and $\mathrm{K}$ bands. In these regions, effective areas of the spectrum where emission lines can be observed without contending with strong night-sky lines are limited. In the coming years, the multiplex advantage of KMOS at VLT will allow us to compile significantly larger samples of galaxies studies with IFUs at $\mathrm{z}>1$. But the collecting area of the current generation of telescopes and seeing limited performance will still limit the size of these samples, since objects will have to be selected such that their emission lines do not overlap with strong night-sky lines or strong atmospheric absorption; these observations already take several hours on such telescopes. Last but not least, current distant 3D surveys at $\mathrm{z}>2$ contain galaxies selected in a variety of ways (e.g., BzK, Lyman-Break galaxies, etc.), which might result in non-trivial and perhaps uncontrollable biases.

EAGLE on the E-ELT will be a decisive step in removing these limitations. The huge collecting area of the E-ELT and moderate spectral resolution and high multiplex of EAGLE will allow us to select galaxies with a less constrained set of redshifts or colour pre-selection. Potentially, such a combination will allow us to observe all galaxies (down to a limiting magnitude) at almost any redshift, which is the only method to obtain a truly representative, robust sample of galaxies. 
In an first example observing programme, EAGLE will enable us to conduct a broad shallow survey of galaxies, using diagnostic rest-frame optical emission lines from $\mathrm{z} \sim 0.5$ to 5 , principally designed to obtain their kinematics, emission line and continuum morphologies, and clues to the sources of ionization of their ISM. These observations will take about 8-10 hours per field and will observe several hundred galaxies. This part of the programme will take about 100 hours in total. For sources in most favorable redshift ranges of 1.2-1.7, 2-2.6, and 3-3.6, the rest-frame optical emission lines are available in three bands (only two for the highest redshift range listed). In these ranges, we can observe the important diagnostic lines of [OII], [OIII], [NII], $\mathrm{H} \alpha$, and $\mathrm{H} \beta$ as well as other important lines. Such a program will require tens of hours per field of integration time. For 20 galaxies in each redshift range will therefore require 240 hours ( 30 hours per band). Such deep integrations will enable us to investigate their spatially-resolved metallicities and ionization mechanisms and provide a detailed understanding the physical properties of the warm ionized gas in distant galaxies [21].

Current spectroscopic surveys already provide us with redshift catalogues from which EAGLE targets can be drawn. However, these surveys do not have the necessary spectroscopic completeness over several fields of $20 \times 20 \mathrm{arcmin}^{2}$ in size or greater. Deeper, wider surveys could be undertaken with VIMOS but we emphasize the need for good completeness as current surveys generally do not have the requisite target densities down to faint magnitudes/low emission equivalent-widths. ALMA may play a role in this by surveying fields with broad-band receivers in order to obtain redshifts and dynamics of distant galaxies, especially the dusty, optically faint galaxies. Also, the multi-slit mode of JWS T-NIRSPEC could provide sufficient target densities and high completeness necessary for our proposed surveys.

\section{References}

1. Evans, C. et al., these proceedings, arXiv:0909.1748

2. Rousset, T. et al., these proceedings

3. Schnetler, T. et al., these proceedings

4. Morris, T. et al., these proceedings

5. Vidal, T. et al., these proceedings

6. Paumard, T. et al., these proceedings

7. Hammer, F., Lehnert, M., Puech, M., Flores, H., \& Liang, Y.-C. 2006, The Messenger, 123, 41

8. Puech, M., Hammer, F., Flores, H., Neichel, B., Yang, Y., \& Rodrigues, M. 2007, A\&A, 476, L21

9. Puech, M., Flores, H., Lehnert, M., Neichel, B., Fusco, T., Rosati, P., Cuby, J.-G., \& Rousset, G. 2008, MNRAS, 390, 1089

10. Epinat, B., Amram, P., Balkowski, C., \& Marcelin, M. 2009, arXiv:0904.3891

11. Puech, M., Rosati, P., Toft, S., Neichel, B., \& Fusco, T. 2008b, SPIE Proc., Vol. 7014, 203

12. Puech, M., Rosati, P., Toft, S., et al., 2009, MNRAS, submitted.

13. Elmegreen, B. G., Elmegreen, D. M., Chandar, R., Whitmore, B., \& Regan, M. 2006, ApJ, 644, 879

14. Elmegreen, B. G., \& Elmegreen, D. M. 2005, ApJ, 627, 632

15. Bournaud, F., \& Elmegreen, B. G. 2009, ApJL, 694, L158

16. Dekel, A., et al. 2009, Nature, 457, 451

17. Yang, Y., et al. 2008, A\&A, 477, 789

18. Forster Schreiber, N. M., et al. 2009, arXiv:0903.1872

19. Law et al., ApJ, 2009, 697, 2057

20. Wright et al., ApJ, 699, 421

21. Lehnert, M. D., Nesvadba, N. P. H., Tiran, L. L., Matteo, P. D., van Driel, W., Douglas, L. S., Chemin, L., \& Bournaud, F. 2009, ApJ, 699, 1660 